\title{
Directing evolution in cells
}

\author{
A CRISPR-Cas9-based approach generates libraries of mutant proteins targeted to specific subcellular \\ compartments for in situ directed evolution.
}

$\mathrm{D}$ irected evolution is the process of mutagenizing and screening biomolecules, such as RNA and proteins, to select for specific desired properties. This process has yielded a wealth of results that encompass better enzymes, widely used fluorescent proteins, thermostable proteins and beyond.

Mutlu Erdogan, Arne Fabritius and colleagues in the laboratory of Oliver Griesbeck at the Max Planck Institute of Neurobiology near Munich, Germany, were interested in developing improved methods for directed evolution of proteins. "We, like many others, had been relying on in vitro selection or microbial expression systems for our protein engineering work," recalls Griesbeck. "While these approaches are low cost and allow screening large numbers of variants in a protein library, they often do not constitute the appropriate context for evaluating the performance of a library member."

Griesbeck's research group has a long-standing interest in engineering improved fluorescent protein-based probes, and they sought a method for doing directed evolution in mammalian cells that was efficient in terms of library construction, was compatible with large libraries, and would also allow the screening of proteins targeted to specific subcellular locations. Their method exploits the fact that DNA repair occurring at double-stranded DNA breaks can introduce considerable genetic diversity to generate libraries in the context of living mammalian cells. As Griesbeck explains, "we use Cas9 to introduce targeted double-strand breaks in a region of DNA coding for a protein of interest. The double-strand break triggers cellular repair systems to fix the double-strand break. Via these DNA repair processes, diversity is introduced in the protein-coding region, which finally results in a diversified protein."

More specifically, the researchers generate stable mammalian cell lines harboring a single copy of the gene encoding the protein of interest and then subject the target gene to CRISPR-Cas9-based mutagenesis. They guide the mutagenesis by the choice of cut sites and with the use of exogenously added, specifically designed
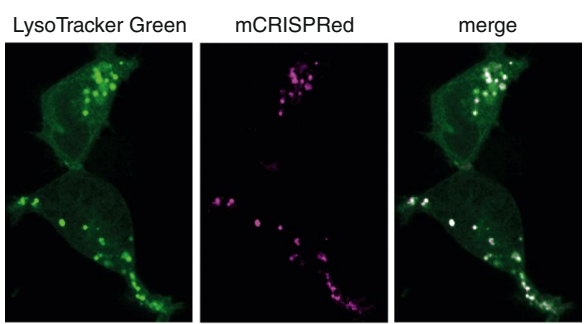

Lysosomes in mammalian cells labeled with LysoTracker (green, left) and mCRISPRed (red, middle). The right panel shows the red and green images merged. Adapted with permission from Erdogan, M. et al. Cell Chem. Biol. doi:10.1016/ j.chembiol.2020.02.004, 2020, Elsevier.

short single-stranded DNA oligonucleotides to create variants. Mutagenesis is followed by screening for clones with desired features. As a proof-of-principle experiment, they showed that their strategy could be used to restore a truncated reading frame between two fluorescent proteins of interest. Subsequent RNA-seq analysis revealed that a combination of homology-directed recombination (HDR) and non-homologous end joining (NHEJ) was used for this repair.

The researchers then optimized a fluorescent protein for labeling lysosomes. Lysosomes are challenging to label because their $\mathrm{pH}$ is typically much lower than the $\mathrm{p} K_{\mathrm{a}}$ of a fluorescent protein. The team targeted the construct they wanted to mutagenize to the lumen of the lysosome via fusion to a lysosomal resident protein. Their construct was a fusion of two fluorescent proteins: the red fluorescent protein mRuby 3 and the blue fluorescent protein mTagBFP2. Upon expression in the lumen of lysosomes, mRuby $3 \mathrm{had} \mathrm{dim}$ fluorescence while mTagBFP2, which is known to be less compromised at low $\mathrm{pH}$, showed stronger signal. The authors focused mutagenesis on regions surrounding the mRuby 3 chromophore and screened via fluorescence-activated cell sorting for mutants with increased red fluorescence at low $\mathrm{pH}$.

Their screen led to the discovery of mCRISPRed, which brightly labels lysosomes. Compared to mRuby3, mCRISPRed has a much lower $\mathrm{p} K_{\mathrm{a}}$ of 2.1, which explains its robustness under acidic conditions. However, the overall brightness at neutral $\mathrm{pH}$ was diminished. mCRISPRed also has a long Stokes shift due to a roughly 100 -nm blueshift in the absorbance maximum, which indicates that the mCRISPRed chromophore is protonated and undergoes excited-state proton transfer, like many fluorescent proteins with a long Stokes shift.

Despite the successful applications of their approach, Griesbeck notes that they faced challenges. "On the practical side, one big challenge was to push the balance of cellular repair outcomes into the right direction." He says that compared to NHEJ, HDR "is much more powerful and flexible in the type of mutagenesis it enables," and "thus, a big challenge was to enrich cells and library variants repaired via HDR." He also shares that improvements to the approach will involve increasing the percentage of HDR-repaired proteins in the library, as well as simultaneously diversifying two separate regions of a target gene that are far apart in the primary sequence.

In terms of next steps, Griesbeck says that the approach should be applied "to a number of protein engineering projects where mammalian cell context is essential." He also notes that speed, efficiency and library quality are practical points to consider when trying this approach. The approach takes an important step forward for identifying proteins that function well in specific cellular contexts, and represents an exciting use of gene-editing technology. As Griesbeck shares, "Protein diversification may become another great application for CRISPR/Cas."

\section{Rita Strack}

Published online: 5 May 2020

https://doi.org/10.1038/s41592-020-0829-5

Research paper

Erdogan, M. et al. Targeted in situ protein

diversification and intra-organelle validation in mammalian cells. Cell Chem. Biol. https://doi. org/10.1016/j.chembiol.2020.02.004 (2020). 